\title{
Calibração do modelo de consumo de combustível do simulador AIMSUN com dados coletados via OBD
}

\author{
Arthur Theodoro Marinho ${ }^{1}$, Renan Artur Lopes Eccel ${ }^{2}$, Rodrigo Castelan Carlson ${ }^{3}$, \\ Werner Kraus Junior ${ }^{4}$
}

1Universidade Federal de Santa Catarina, Pós-Graduação em Engenharia de Automação e Sistemas, ufsc.arthur@gmail.com 2Universidade Federal de Santa Catarina, Pós-Graduação em Engenharia de Automação e Sistemas, renan.eccel@gmail.com ${ }^{3}$ Universidade Federal de Santa Catarina, Pós-Graduação em Engenharia de Automação e Sistemas, rodrigo.carlson@ufsc.br ${ }^{4}$ Universidade Federal de Santa Catarina, Pós-Graduação em Engenharia de Automação e Sistemas, werner.kraus@ufsc.br

\section{Recebido:}

5 de junho de 2017

Aceito para publicação:

24 de agosto de 2018

Publicado:

31 de agosto de 2018

Editor de área:

Helena Beatriz Cybis

\section{Palavras-chaves:}

OBD,

Modelagem,

Consumo de Combustível,

Microssimulação de Tráfego.

\section{Keywords:}

OBD,

Modeling,

Fuel consumption,

Traffic Microsimulation

DOI:10.14295/transportes.v26i6.1393

\begin{abstract}
RESUMO
Dados coletados por meio da interface de diagnóstico de bordo (On-Board Diagnostics - OBD), presente nos automóveis nacionais fabricados a partir de 2009, foram usados na calibração de modelo microscópico de consumo de combustível do simulador AIMSUN. Para a calibração dos parâmetros do modelo de consumo, mediu-se a velocidade instantânea, o fluxo de ar de admissão e o teor de oxigênio na mistura ar/combustível. Calibrou-se o modelo de consumo do simulador seguindo a abordagem modal, usando regressão para os modos de aceleração e velocidades constantes e média simples para os modos de marcha lenta e desaceleração. Para validar o modelo, os consumos de combustível medido e estimado foram comparados em ciclos de condução em áreas urbanas. Os resultados indicam a adequação do método para avaliação do consumo de combustível por veículos em malhas viárias urbanas. Além disso, o modelo calibrado com dados do OBD de quatro tipos de veículos foi usado no estudo por simulação de cenários atual e futuro no contexto de projeto de ampliação viária. Por meio de abordagem comparativa entre cenários, mostra-se que a estimação com parâmetros calibrados produz indicadores distintos e mais confiáveis em relação aos obtidos com os parâmetros originais do simulador.
\end{abstract}

\section{ABSTRACT}

Data collected through the On-Board Diagnostics (OBD) interface, present in domestic cars manufactured since 2009, was used in the calibration of the microscopic fuel consumption model of the AIMSUN simulator. Calibration of the consumption model required data for instantaneous velocity, intake air flow and oxygen content in the air/fuel mixture. The simulator consumption model was calibrated following the modal approach using regression for acceleration and constant velocities modes and simple mean for idle and deceleration modes. To validate the model, measured and estimated fuel consumption in driving cycles in urban areas were compared. The results indicate the suitability of the method to evaluate fuel consumption by vehicles in urban road networks. In addition, the OBD-calibrated model of four types of vehicles was used in the simulation study of current and future scenarios in the context of a road expansion project. By means of a comparative approach between scenarios, it is shown that the estimation with calibrated parameters produces different and more reliable indicators than those obtained with the original parameters of the simulator.

\section{INTRODUÇÃO}

Simuladores de tráfego representam o comportamento do tráfego de veículos para produção de indicadores de desempenho do sistema viário analisado. Dentre esses indicadores, o consumo de combustível e as correspondentes emissões têm sido objeto de crescente interesse de pesquisadores e dos gestores de áreas urbanas, pois permitem avaliar o resultado de operações de 
transportes em termos de efeitos ambientais. Assim, obtém-se elementos de avaliação além de medidas de desempenho usuais como atraso e número de paradas. Em particular, o consumo de combustível indica a produção de $\mathrm{CO}_{2}$ pelo veículo (An et al., 2011) principal agente contribuinte para o aumento do efeito estufa.

O consumo de combustível para avaliação de sistemas via simulação é encontrado em estudos de controle de tráfego e otimização (Osorio e Nanduri, 2015), custo operacional (Akçelik e Besley, 2003) e emissões de $\mathrm{CO}_{2}$ (Akçelik et al., 2012). Dada a importância desse indicador, o objetivo deste trabalho é estabelecer um método para calibrar e validar modelos de consumo de combustível em microssimulador de tráfego. Para a demonstração dos resultados é usado o modelo de consumo de combustível do simulador de tráfego AIMSUN.

A calibração de modelos de simulação para o consumo de combustível de veículos requer dados operacionais tais como a velocidade instantânea, o consumo instantâneo e o modo de operação do veículo. Obter dados para a calibração é, portanto, uma tarefa potencialmente onerosa em termos de instrumentação e tipos de ensaios a realizar. Entretanto, o avanço tecnológico dos veículos automotores tem facilitado a tarefa de obtenção dos dados por meio de instrumentação embarcada. Nesse sentido, destaca-se a presença, nos veículos fabricados no Brasil desde 2009, de dispositivo denominado diagnóstico de bordo ou OBD (da sigla para o inglês On-Board Diagnostics) (Baltusis, 2004). O OBD consiste de um conector padrão localizado sob o painel de veículos e fornece um protocolo de comunicação padronizado para acesso às variáveis de operação, dentre as quais aquelas associadas ao consumo de combustível.

O OBD opera como instrumentação de medição embarcada de variáveis do veículo. Neste trabalho, o OBD é utilizado para medir o consumo de combustível em quatro tipos de veículos, bem como as demais variáveis associadas ao regime de operação ao longo de ciclos de condução. Para fins do estudo, devem ser considerados o modelo matemático de consumo de combustível do simulador, as formas de aquisição de dados para estimação do modelo, o registro dos ciclos de condução e método para calibração e validação do modelo.

A abordagem empregada tem as vantagens de ser compacta, precisa e de baixo custo - menos de US\$100,00 para aquisição do data logger e licença do aplicativo, que compõem o sistema de aquisição de dados em um dispositivo tablet ou smartphone com GPS integrado. Em contraste, os analisadores de emissões denominados PEMS (Portable Emissions Measurements Systems) (Franco et al., 2013) necessitam de calibração periódica, têm alto custo e instalação dificultada no veículo. Por isso, estudos de grande escala se tornam infactíveis. Como consequência, pouco se sabe sobre o efeito das condições do mundo real sobre as emissões durante o período de condução (Thibault et al., 2016).

Na Seção 2, apresenta-se o modelo de consumo de combustível do simulador microscópico AIMSUN e sua aplicação. Em seguida, na Seção 3, discutem-se os métodos usados para aquisição de dados e de calibração do modelo. Na Seção 4, os resultados obtidos da calibração e validação são apresentados. A implementação dos resultados da calibração é apresentada para um estudo de caso na Seção 5, com considerações finais na Seção 6.

\section{MODELOS DE CONSUMO DE COMBUSTÍVEL PARA MICROSSIMULAÇÃO DE TRÁFEGO}

Modelos de consumo de combustível podem ser macroscópicos ou microscópicos (Nanduri, 2013). Os modelos macroscópicos estimam o consumo baseado na velocidade e aceleração médias na via, isto é, de maneira agregada. Modelos microscópicos usam velocidade e aceleração 
instantâneas de veículos individuais para estimar o consumo de combustível e são, portanto, potencialmente mais precisos.

A premissa de um modelo elementar microscópico para consumo de combustível é a independência das quantidades de combustível consumidas durante os quatro modos de condução fundamentais, denominados marcha lenta (idle), velocidade constante, desaceleração e aceleração (Akçelik, 1983). Assume-se nesses modelos, chamados de modais, que o consumo total pode ser obtido pela adição dos consumos associados a cada um dos modos independentemente da ordem em que ocorrem.

A abordagem modal foi proposta inicialmente por Watson (1973) para um modelo de emissão de poluentes. Tal trabalho constatou que as taxas de emissões nos gases de escapamento, bem como as taxas de consumo de combustível, podiam ser representadas com precisão em função da velocidade e aceleração. Assim, subdividiu a condução nos quatro modos e, para cada modo, o modelo previa a emissão de poluentes em função das acelerações e velocidades instantâneas. A vantagem deste modelo é a sua simplicidade, generalidade e clareza conceitual, bem como a relação direta com técnicas de modelagem de tráfego (Ahn, 1998).

\subsection{Modelo de consumo de combustível do simulador AIMSUN}

O modelo de consumo de combustível do AIMSUN é classificado como um modelo elementar modal microscópico. 0 modo de condução de cada veículo é identificado a partir dos valores de velocidade e aceleração. Uma vez identificado o modo de condução, aplica-se uma fórmula apropriada para calcular o combustível consumido, conforme descrito a seguir com base em informações do manual do simulador (TSS, 2015).

\subsubsection{Consumo de combustível em marcha lenta}

O modo "marcha lenta" (idle) ocorre com o motor ligado e com velocidade e aceleração iguais a zero. No AIMSUN, o consumo de combustível em marcha lenta, $F_{\mathrm{i}}(\mathrm{mL} / \mathrm{s})$, é considerado constante (TSS, 2015). De fato, de acordo com Ferreira (1982), para veículos nesse modo a taxa de consumo de combustível pode ser assumida como constante. A estimação do consumo em marcha lenta é importante pois representa evento frequente em tráfego urbano como, por exemplo, enquanto o veículo estiver parado em um semáforo (Akçelik, 1983) ou em congestionamento.

Existem alguns sistemas aplicados a automóveis para redução do consumo de combustível como, por exemplo, o Stop-Start, que desliga o motor nos instantes em que o veículo está parado (Owens e Laughlin, 2016). Neste trabalho, não são considerados veículos equipados com essa tecnologia [ver Marinho (2016) para resultados com a tecnologia Stop-Start].

\subsubsection{Consumo de combustível em velocidades constantes (cruzeiro)}

O modelo implementado no simulador AIMSUN para velocidades constantes baseia-se no modelo de consumo instantâneo de combustível apresentado por Akçelik (1983) e é dado por (TSS, 2015):

$$
f=\frac{d F}{d t}=k_{1}\left(1+\frac{V^{3}}{2 v_{\mathrm{m}}^{3}}\right)+k_{2} V,
$$

em que $f(\mathrm{~mL} / \mathrm{s})$ é a taxa de consumo de combustível, $F(\mathrm{~mL})$ é o consumo de combustível, $t(\mathrm{~s})$ é o tempo, $v(\mathrm{~km} / \mathrm{h})$ é a velocidade instantânea, $k_{1}(\mathrm{~mL} / \mathrm{s})$ é a taxa de consumo em marcha lenta, 
$k_{2}(\mathrm{~mL} \cdot \mathrm{h} / \mathrm{km} \cdot \mathrm{s})$ é a taxa de consumo referente à resistência de rolamento, e $v_{\mathrm{m}}(\mathrm{km} / \mathrm{h})$ é a velocidade de consumo mínimo por unidade de distância. Akçelik (1983) define $k_{1}$ na Equação 1 como a taxa de consumo de combustível em marcha lenta; no AIMSUN, $k_{1}$ é um parâmetro livre a ser calibrado.

0 veículo se mantém em modo de condução de velocidade constante enquanto sua aceleração ou desaceleração não excederem a aceleração de tolerância, $a_{\mathrm{t}}\left(\mathrm{m} / \mathrm{s}^{2}\right)$, isto é, enquanto $-a_{\mathrm{t}} \leq a \leq a_{\mathrm{t}}$ e $v>0 \mathrm{~km} / \mathrm{h}$.

\subsubsection{Consumo de combustível durante a aceleração}

No AIMSUN, o combustível consumido durante a aceleração, $F_{\mathrm{a}}(\mathrm{mL} / \mathrm{s})$, é determinado por (TSS, 2015):

$$
F_{\mathrm{a}}=c_{1}+c_{2} \mathrm{aV},
$$

em que $c_{1}(\mathrm{~mL} / \mathrm{s})$ e $c_{2}\left(\mathrm{~mL} \mathrm{~s}^{2} / \mathrm{m}^{2}\right)$ são constantes a serem calibradas e $a\left(\mathrm{~m} / \mathrm{s}^{2}\right)$ e $v(\mathrm{~m} / \mathrm{s})$ são a aceleração e a velocidade instantâneas do veículo, respectivamente. Na Equação 2, o produto $c_{2} a v$ define a inércia do veículo (massa em translação e rotativa). 0 produto da velocidade $\mathrm{e}$ aceleração representa uma indicação da demanda de potência requerida pelo motor (Hickman, 1988). Para ser considerado no estado de aceleração, o veículo deve ter $a>a_{\mathrm{t}}$ e $v>0 \mathrm{~m} / \mathrm{s}$.

\subsubsection{Consumo de combustível durante a desaceleração}

A taxa de consumo de combustível durante a desaceleração, $F_{\mathrm{d}}(\mathrm{mL} / \mathrm{s})$, é considerada constante no AIMSUN. Nesse modo, o veículo parte de uma determinada velocidade inicial constante e permanece no modo até atingir o repouso. Nos veículos modernos, a estratégia de controle da injeção de combustível de cut-off, é utilizada nesse modo e resulta no corte do combustível (Alessandrini et al., 2004). 0 veículo deve ter $a<-a_{\mathrm{t}}$ e $v>0 \mathrm{~m} / \mathrm{s}$ para estar nesse modo.

\subsection{Aplicação do modelo de consumo de combustível do AIMSUN}

O AIMSUN implementa o modelo modal para o consumo de combustível verificando a cada passo de simulação os valores de aceleração e de velocidade instantâneas de cada veículo. Com estes, determina o modo de condução em vigor, aplicando a equação respectiva de cálculo do consumo de combustível. Neste trabalho, a aceleração de tolerância que define cada modo de condução foi definida como 0,278 m/s² (Akçelik et al., 2012).

\section{MÉTODOS DE AQUISIÇÃO DE DADOS E DE CALIBRAÇÃO DO MODELO}

Depreende-se da apresentação do modelo (Seção 2), que três dados são necessários para o processo de calibração: a velocidade e a aceleração, que são variáveis do modelo e são também necessárias para determinar o modo de condução do veículo, e o consumo de combustível. A seguir, após a descrição do OBD, apresenta-se como esse dispositivo pode ser usado para a coleta desses dados.

\subsection{Diagnóstico de bordo (OBD)}

Nas décadas de 1970 e 1980, os fabricantes de automóveis passaram a utilizar meios eletrônicos para controlar as funções do motor e diagnosticar problemas. Em função disso, em 1988 a Sociedade de Engenheiros Automotivos (hoje SAE International) definiu um conector padrão e um conjunto de sinais de teste de diagnóstico que compõem o On-Board Diagnostics (OBD) ou Diagnóstico de Bordo (Baltusis, 2004). Os padrões do OBD especificam o tipo de conector e sua 
pinagem, o protocolo de sinais e o formato da mensagem, além de fornecer uma lista dos parâmetros disponíveis para monitorar os dados do motor, chassi, carroceria e acessórios dos automóveis. No Brasil, o sistema OBD-BR1 tornou-se obrigatório a todos os veículos novos comercializados a partir de janeiro de 2009. Um ano depois o OBD-BR2 foi implementado e em janeiro de 2011 atingiu 100\% da frota de carros novos (CONAMA, 2004). Neste trabalho, o uso da sigla OBD refere-se ao padrão OBD-BR2.

\subsection{Sistema de aquisição de dados}

O processo de aquisição de dados, calibração e validação do modelo são apresentados no diagrama da Figura 1(a). 0 sistema de aquisição utiliza dois sensores automotivos para estimar o consumo de combustível de um veículo - sensor de oxigênio e sensor de fluxo de massa de ar e um sensor de velocidade instantânea do veículo. A EECU (da sigla para o inglês Engine Electronic Control Unit) os interpreta e disponibiliza na rede de comunicação do veículo, podendo ser obtidos a partir da interface OBD. Para isso, neste trabalho, optou-se pela utilização de um dispositivo data logger Vgate Scan ELM 327, com capacidade de comunicação Bluetooth. 0 aplicativo Torque Pro v.1.8.154 foi utilizado para decodificar as mensagens transmitidas pelo data logger. 0 aplicativo grava as informações solicitadas em forma de um log de dados em um tablet com sistema operacional Android. Os dados coletados são transmitidos para um computador via rede $\mathrm{Wi}-\mathrm{Fi}$, em formato .csv (comma separated value), compatível com diversas planilhas eletrônicas. A seguir, os dados são tratados de forma a manter apenas as informações relevantes para a calibração do modelo. 0 arquivo resultante é importado no programa MATLAB $2014 \mathrm{em}$ que a função correspondente do modo de condução em que o veículo se encontra é utilizada para obter os parâmetros do modelo. Com os parâmetros obtidos da calibração, é possível comparar a saída do modelo com valores reais obtidos em um teste de campo como forma de validar a calibração.
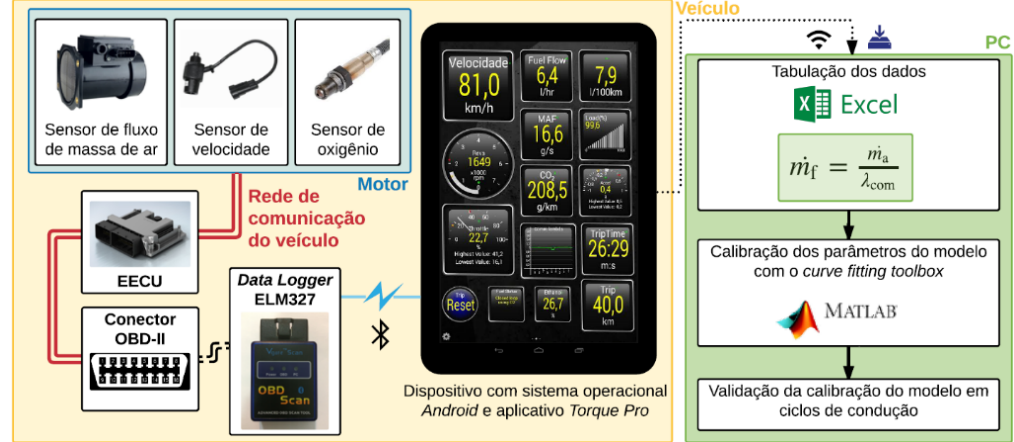

Figura 1. Elementos do processo de aquisição de dados e de calibração e validação do modelo de consumo de combustível

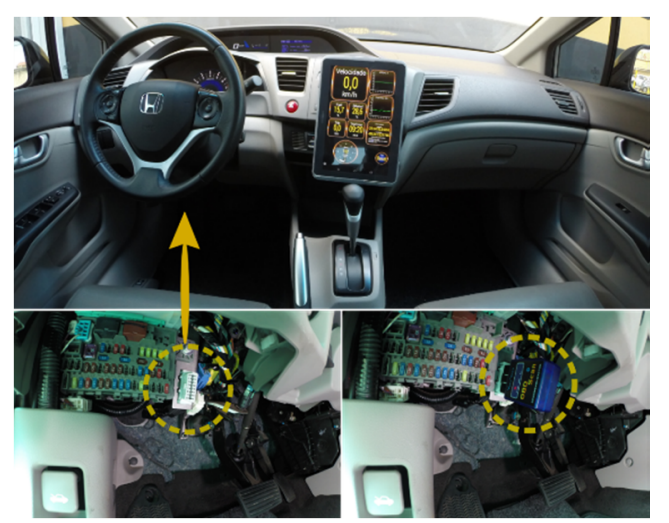

Figura 2. Localização do conector OBD no painel de um veículo Honda Civic ano 2013 
A Figura 1 superior, mostra o aplicativo Torque Pro sendo executado no tablet sobre o painel de um veículo Honda Civic ano 2013. A posição do conector OBD nesse veículo (branco) e o data logger conectado ao terminal OBD aparecem nas imagens inferiores. Tipicamente o conector fica posicionado próximo à central de fusíveis sob o painel de instrumentos.

\subsection{Velocidade, aceleração e consumo de combustível}

A velocidade instantânea do veículo é obtida diretamente pelo OBD. A aceleração não é fornecida, sendo estimada conhecendo-se a taxa de amostragem e dois valores sucessivos de velocidade (ver Seção 3.4). 0 consumo de combustível também não é fornecido diretamente pelo OBD e precisa ser estimado a partir de outros dados. Para isso é necessário conhecer o controle da razão ar/combustível que entra no motor, descrito a seguir.

O funcionamento de um motor a combustão depende da mistura de ar e combustível, cuja razão é controlada pela injeção de combustível no motor. Quando o motorista aciona o pedal do acelerador, ele de fato regula a quantidade de ar que entra no motor. A partir da medida da massa de ar obtida de um sensor de fluxo de massa de ar, o sistema de controle determina a quantidade de combustível a ser injetada, comandando um valor instantâneo de lambda $\left(\lambda_{\text {com }}\right)$, para que a razão ar/combustível seja estequiométrica (razão $\mathrm{Ar} /$ Gasolina: $\lambda_{\text {esteq }}=14,7$; $\mathrm{Ar} /$ Etanol: $\left.\lambda_{\text {esteq }}=9,1\right)$.

0 controle da razão ar/combustível é também chamado de controle lambda, pois é realizado em função da razão ar/combustível normalizada dada por (Ulsoy et al., 2012):

$$
\lambda=\frac{m_{\mathrm{a}}}{m_{\mathrm{f}}} \cdot \frac{1}{\lambda_{\text {esteq }}}
$$

em que $\dot{m}_{\mathrm{a}}$ (g/s) é o fluxo de massa de ar que entra no motor e $\dot{m}_{\mathrm{f}}(\mathrm{g} / \mathrm{s}$ ) é o fluxo de massa de

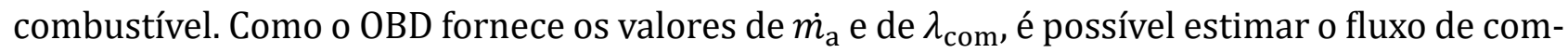
bustível instantâneo injetado no motor a partir da Equação 3 como (Fonseca et al., 2012):

$$
m_{\mathrm{f}}=\frac{\dot{m}_{\mathrm{a}}}{\lambda_{\text {com }} \cdot \lambda_{\text {esteq }}}
$$

Experimentos realizados por Fonseca et al. (2012) mostraram um erro de apenas 3\% com o uso dessa técnica em relação a medidas de referência da massa de combustível no tanque do automóvel.

A gasolina E27 comercializada no Brasil é uma mistura de gasolina e etanol, com a densidade tipicamente de $0,75 \mathrm{~g} / \mathrm{cm}^{3}$ se a proporção etanol gasolina estiver exata (FISPQ 2014). Desde março de 2015, a concentração de $27 \%$ de etanol é determinada por lei (CIMA, 2015). Para estimar o fluxo de combustível neste trabalho usou-se a massa específica igual a $0,75 \mathrm{~g} / \mathrm{cm}^{3}$. Nesse caso, a fórmula para obtenção do fluxo de combustível $\dot{f}_{\mathrm{f}}(\mathrm{mL} / \mathrm{s})$ a partir do fluxo de massa de combustível $\dot{m}_{\mathrm{f}}(\mathrm{g} / \mathrm{s})$ é definida como:

$$
\dot{f}_{\mathrm{f}}=\frac{\dot{m}_{\mathrm{f}}}{0,75}
$$




\subsection{Coleta de dados}

A coleta de dados foi feita de duas maneiras. Na primeira foi considerado o consumo de combustível em testes pré-definidos dos modos de condução, isto é, o veículo foi colocado em condições de funcionamento que correspondem a cada modo de condução, respeitando a aceleração de tolerância apresentada na Seção 2.2. A segunda é a coleta de dados em ciclos de condução, em que os resultados da calibração são aplicados no modelo, a fim de comparar os resultados obtidos ao longo de um ciclo para os consumos medidos e estimados. Todos os dados foram coletados com taxa de $1 \mathrm{~Hz}$ (1 amostra por segundo), ou seja, $\Delta t=1 \mathrm{~s}$ (Akçelik, 2012).

\subsubsection{Coleta de dados em testes pré-definidos de cada modo de condução}

Nesta coleta de dados, coloca-se o veículo para operar em cada um dos modos de condução. No modo de marcha lenta, o veículo permanece parado por um certo período. No modo de velocidade constante, o veículo opera em velocidade constante por um certo período, para diferentes valores de velocidade. No modo de aceleração, o veículo move-se do repouso até uma velocidade final desejada com aceleração constante para vários valores de velocidade final e vários valores de aceleração. De maneira análoga, para o modo de desaceleração faz-se o inverso com velocidade inicial diferente de zero até atingir o repouso. Em todos os casos o experimento foi feito com o motor quente. Para todos os modos de condução, os dados de consumo de combustível foram obtidos em uma via plana. Os dados obtidos nos testes que representam os quatro modos de condução foram utilizados na calibração do modelo de consumo de combustível do AIMSUN e os resultados apresentados na Seção 4.1.

\subsubsection{Coleta de dados em ciclos de condução}

Ciclo de condução (driving cycle) é uma sequência de regimes (modos) de operação com o intuito de representar um padrão típico de condução amplamente usado nos estudos de emissões (Lai et al., 2013). Os ciclos de condução são normalmente definidos em termos da velocidade do veículo e seleção de marchas em função do tempo. No Brasil, o ciclo de condução adotado para determinação de emissões em veículos leves e comerciais leves é o ciclo norte americano conhecido como FTP-75 (Federal Test Procedure 75) (Barlow et al., 2009). Os procedimentos desse ensaio são descritos na NBR 6601 (ABNT, 2012) enquanto que a medição do consumo de combustível é normatizada pela NBR 7024 (ABNT, 2010). Tipicamente, os testes em veículos de passeio e comerciais leves são realizados em laboratório em um dinamômetro de chassi. Nesses testes, as rodas do veículo permanecem em contato com rolos ajustados para simular a resistência aerodinâmica e as perdas por atrito. A amostragem das emissões dos gases de escape é então realizada conforme o veículo progride no ciclo de condução pré-definido. Aproximadamente $0,3 \%$ de todos os veículos produzidos no Brasil devem passar por teste de emissões (Gomes, 2016).

A seleção de um ciclo de condução, projetado para representar padrões de condução do veículo em determinada região é elemento essencial de testes de consumo e emissões. No entanto, os ciclos de condução podem ser muito diferentes de como os veículos são dirigidos de fato, resultando em lacunas entre os níveis de economia de combustível certificados e níveis de economia de combustível reais (Lai et al., 2013). De fato, estudos sugerem que os testes sejam realizados em condições reais de tráfego (André et al., 2005; Liu et al., 2016). 


\subsection{Técnica de calibração do modelo de consumo de combustível do AIMSUN}

Diversas maneiras podem ser adotadas como técnica para a calibração do modelo, como por exemplo, via regressão, ou via instruções do manual do usuário ou ainda com dados de um ciclo de condução real. A técnica de calibração do modelo adotada neste artigo seguiu a abordagem modal, com testes pré-definidos para cada modo. Para os modos de velocidades constantes e aceleração foi adotada a técnica de regressão para a obtenção dos parâmetros de simulação. Para os modos marcha lenta e desacelerações, a taxa de consumo de combustível foi calculada em forma de média simples das amostras.

\section{RESULTADOS DA CALIBRAÇÃO E VALIDAÇÃO DO MODELO}

A calibração de um modelo de consumo de combustível é válida para um tipo específico de veículo. Com efeito, para que haja uma melhor avaliação do consumo de combustível global em simulação, vários tipos de veículos/motorização devem ser considerados na calibração do modelo. A calibração e validação do modelo de consumo de combustível do AIMSUN foram realizadas para quatro veículos de vários pesos e tamanhos de motor. Nesta seção, serão apresentados os resultados da calibração e validação do modelo para um veículo Honda Civic motor 1.8 L. Os resultados para os demais veículos, Ford Fiesta motor 1.0 L, Volkswagen Saveiro motor 1.6 L e Ford Fusion motor 2.4 L são apresentados por Marinho (2016) e são utilizados como entrada no simulador para este trabalho. Juntos, os quatro veículos cobrem uma ampla faixa de motorizações, representativas da atual tecnologia dos motores de combustão interna que compõem o tráfego de veículos nacionais de passeio.

\subsection{Resultados com dados dos testes de cada modo de condução}

Para o modo marcha lenta, a taxa de consumo de combustível foi estimada por meio de uma média simples das amostras e resultou em $F_{\mathrm{i}}=0,295 \mathrm{~mL} / \mathrm{s} \operatorname{com} \sigma=0,012$. A Figura 2(a) apresenta as amostras de consumo instantâneo de combustível obtidas via OBD na forma de um histograma. As medições foram realizadas durante um período de $200 \mathrm{~s}$ (200 amostras).

Para a calibração do modo de aceleração foram usadas acelerações a partir do repouso $(v=$ $0 \mathrm{~km} / \mathrm{h}$ ) até velocidades finais $v_{\mathrm{f}} \in\{25,50,75,100\} \mathrm{km} / \mathrm{h}$. Foram feitas quatro coletas para cada velocidade final com diferentes taxas de aceleração. A Figura 2(b) mostra a calibração do modelo e os valores de $c_{1}$ e $c_{2}$ (Equação 2) obtidos por regressão linear. Qualitativamente, percebe-se grande dispersão dos dados, refletidos no baixo valor de $R^{2}=0,4493$.

Para o modo velocidade constante, foram considerados dados de 30 amostras para cada valor de velocidade, a intervalos de $5 \mathrm{~km} / \mathrm{h}$, entre 10 e $120 \mathrm{~km} / \mathrm{h}$. A Figura 2(c) apresenta os valores de consumo e os valores dos coeficientes $k_{1}, k_{2}$ e $v_{\mathrm{m}}$ resultantes da regressão pelo método dos mínimos quadrados não linear para o modelo da Equação 1, especificada no curve fitting Matlab. 0 modelo é identificado por C-CB (para $v$ constante). A métrica de $R^{2}$ foi obtida através da soma dos quadrados das diferenças entre a média e cada valor observado. 0 coeficiente de determinação $R^{2}=0,9995$ sugere que a Equação 1 teve um bom ajuste aos dados de consumo de combustível, obtidos via OBD em mL/s. Observa-se na Figura 2(c) que, mesmo mantendo-se a velocidade constante por um certo período de tempo em uma via plana, o consumo de combustível tem uma dispersão maior dos valores em velocidades superiores a $90 \mathrm{~km} / \mathrm{h}$. Isso se dá por conta da influência da resistência do ar no consumo do veículo nessas velocidades. 0 coeficiente de ajuste da taxa de consumo de combustível referente à resistência do ar aparece em função de 
$v_{\mathrm{m}}$ na Equação 1 (Akçelik, 1983). Para validar a calibração do modelo, os valores estimados (C$\mathrm{CB}$ ) foram confrontados com os valores medidos (C-OBD) por outras 30 amostras de cada velocidade constante, a intervalos de $5 \mathrm{~km} / \mathrm{h}$. A Figura 2(d) apresenta o resultado da validação, em que a reta indica o modelo ideal. Para quantificar as diferenças entre os valores medidos e previstos pelo modelo, empregou-se a raiz da média quadrática dos erros (RMSE) (Chai, 2014), calculado com o desvio padrão da diferença entre os valores medidos e estimados.

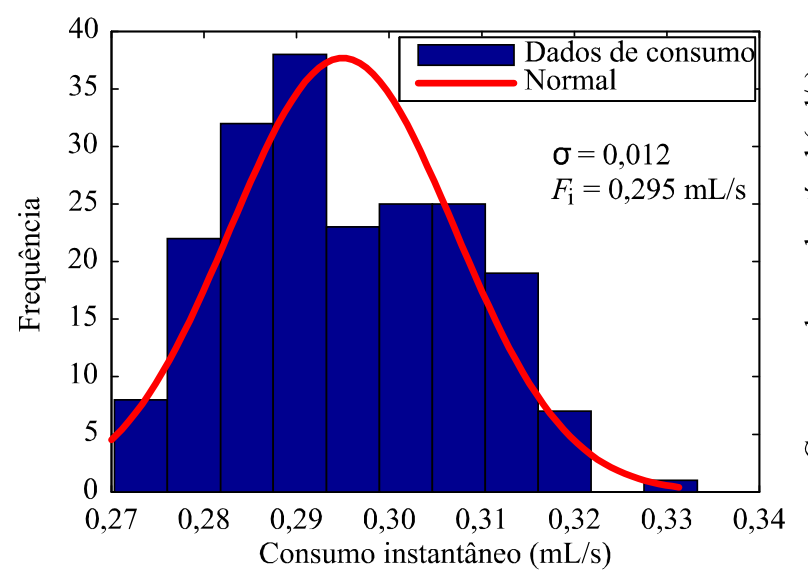

(a)

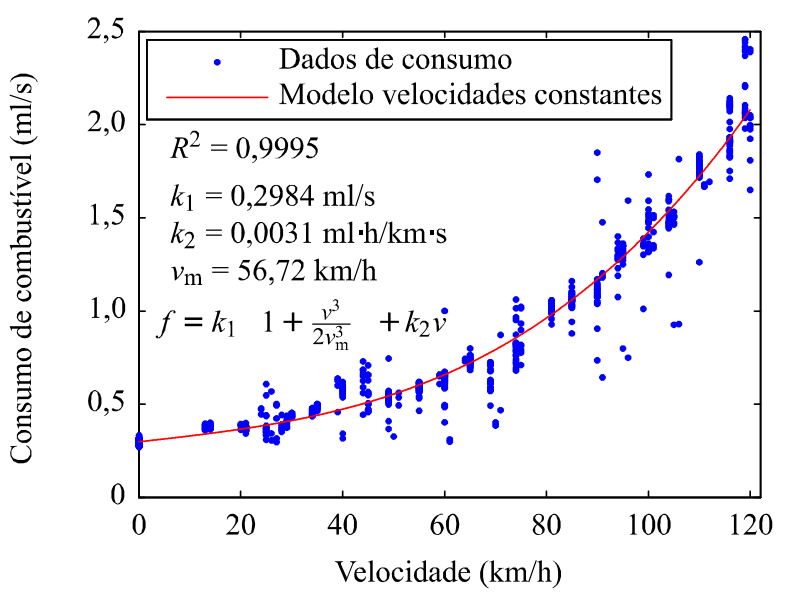

(c)

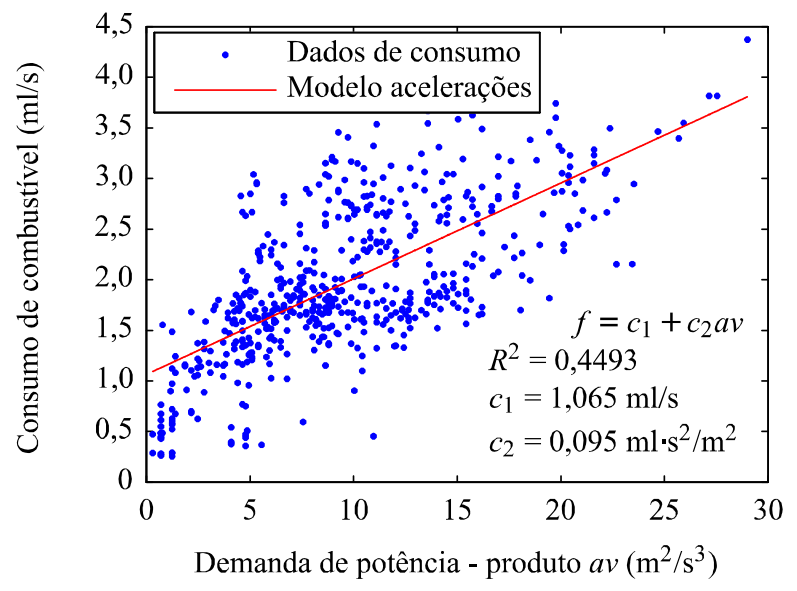

(b)

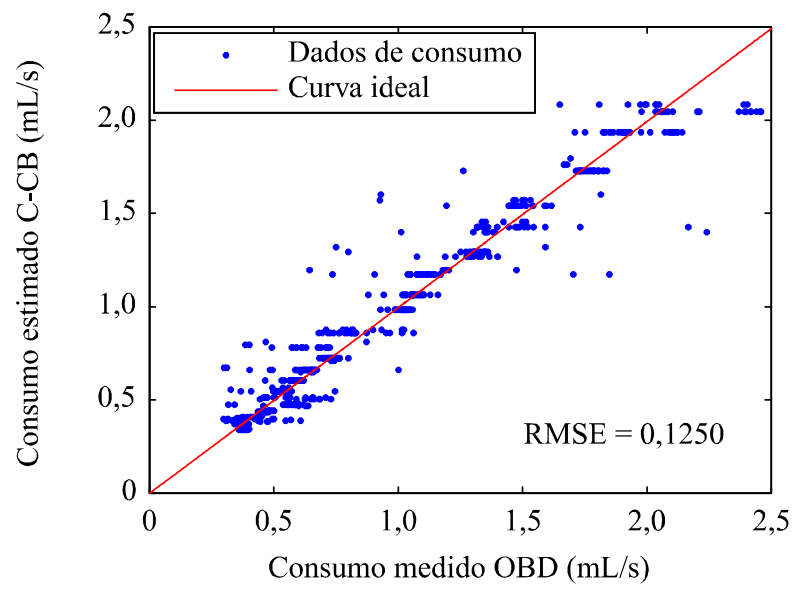

(d)

Figure 2. Resultados da calibração dos modelos de (a) marcha lenta, (b) aceleração, (c) velocidade constante e (d) validação do modelo para velocidades constantes

Para a calibração do modo de desaceleração foram usadas desacelerações a partir de velocidades iniciais $v_{\mathrm{i}} \in\{30,50,80\} \mathrm{km} / \mathrm{h}$ até o repouso $(v=0 \mathrm{~km} / \mathrm{h})$, com quatro rodadas de testes para cada velocidade inicial, com diferentes taxas de desaceleração. Nesse modo, o motorista aciona o pedal do freio do veículo e então, a unidade de controle do veículo reconhece a desaceleração, comanda um valor de $\lambda=2$ e corta a injeção de combustível (cut-off). Após o corte, à medida que o veículo se aproxima de $v=0 \mathrm{~km} / \mathrm{h}$, o motor volta a operar em marcha lenta. A Figura 3(a-c) mostra esses eventos para uma velocidade inicial de $80 \mathrm{~km} / \mathrm{h}$.

A média dos valores de consumo durante várias situações de desaceleração foi usada para estimar a taxa de consumo de combustível nesse modo, que resultou em $F_{\mathrm{d}}=0,185 \mathrm{~mL} / \mathrm{s}$. A taxa em desacelerações é menor do que a taxa em marcha lenta $\left(F_{\mathrm{d}}<F_{\mathrm{i}}\right)$. Isso acontece porque 
durante o período considerado como desaceleração, o veículo passa por dois estados de consumo de combustível: o de cut-off e o de marcha lenta, instantes antes de se aproximar de $v=0$ $\mathrm{km} / \mathrm{h}$.

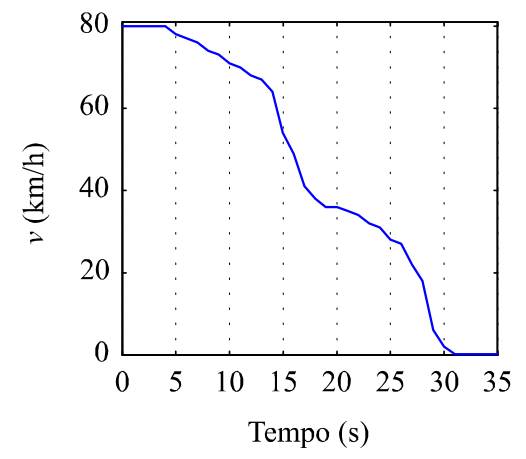

(a)

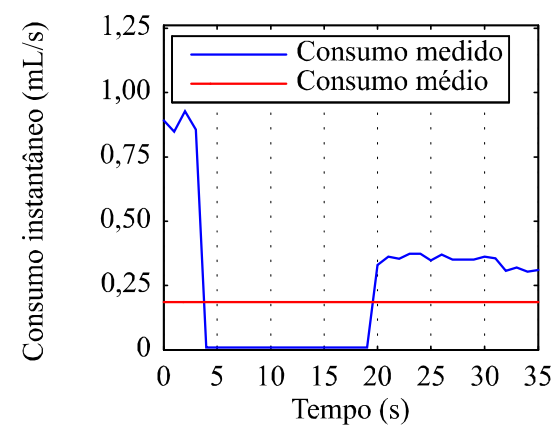

(b)

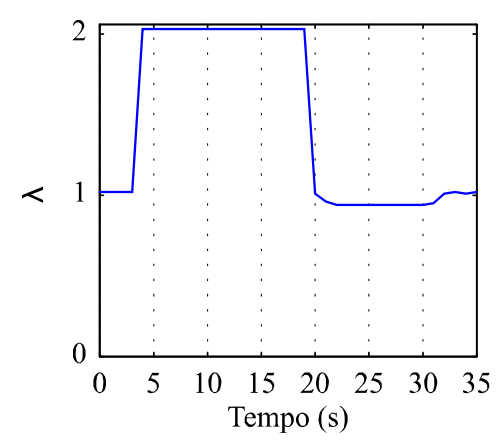

(c)

Figura 3. (a) velocidade, (b) consumo de combustível e (c) variação de lambda durante uma desaceleração

\subsection{Resultados da validação com dados do ciclo de condução}

A partir dos valores dos coeficientes encontrados da calibração do modelo apresentados na Seção 4.1 e utilizando o modelo apresentado na Seção 2, é aplicado um método de validação utilizando ciclos de condução em áreas urbanas. Usaram-se dados coletados em condições de tráfego reais na Av. Beira-mar Norte em Florianópolis/SC. 0 trajeto tem 13,4 km de extensão e 17 semáforos.

Dois cenários foram realizados para comparação dos dados de consumo de combustível medido e estimado. 0 veículo percorreu o trajeto da via por dez vezes. Cinco ciclos foram realizados em condições de pouco tráfego durante a madrugada. Outros cinco ciclos foram realizados com tráfego intenso no início da manhã, quando já existe um certo fluxo de veículos na via, mas não o suficiente para provocar congestionamentos. Para os dois cenários, os ciclos duraram entre 14 e 17 minutos. No cenário com pouco tráfego, o veículo para em nove semáforos e mantémse por períodos mais longos num determinado modo, pois não há influência significativa do tráfego nas medições. 0 cenário foi projetado com velocidades constantes entre semáforos, sempre que possível, para que o veículo parasse no mesmo semáforo em todos os ciclos gerados. No cenário com tráfego intenso, o veículo para em seis semáforos e apresenta mais acelerações e desacelerações por conta da influência dos outros veículos no decorrer do percurso.

A Figura 4 mostra os resultados para um ciclo de cada cenário, com pouco tráfego e com tráfego intenso. São apresentados a velocidade instantânea $v$, a aceleração $a$ estimada, o consumo de combustível (C-OBD) obtido via OBD e via Equação 4 e, por fim, o consumo de combustível estimado usando os valores de $v$ e $a$ como entrada pelo modelo, com parâmetros calibrados dos dados dos testes de cada modo (C-CB). No modo de desaceleração, observa-se nos dois cenários os valores de consumo medidos (C-OBD) iguais a zero (situação de cut-off). Seguindo a mesma abordagem de validação usada no modo velocidade constante (Seção 4.1), o RMSE foi calculado com o desvio padrão da diferença entre os valores medidos estimado. Apesar dos perfis de velocidades, acelerações e consumos de combustível apresentados na Figura 4 serem para apenas um dos ciclos, os valores de RMSE são acumulados para as cinco rodadas de ciclos utilizada de cada cenário. Ver em Marinho (2016) os demais ciclos de condução usados com avaliações individuais de cada ciclo. 
Os resultados do cenário com pouco tráfego forneceram um RMSE melhor do que o cenário com tráfego intenso. Isso se deve à influência do tráfego de outros veículos nas medições e por haver maior número de acelerações e desacelerações durante o ciclo de condução que correspondem aos modos de operações em que o modelo tem pior desempenho. As cores de fundo da figura ilustram o modo de condução do veículo. Vermelho é marcha lenta, azul é velocidade constante, verde é aceleração e amarelo é desaceleração.
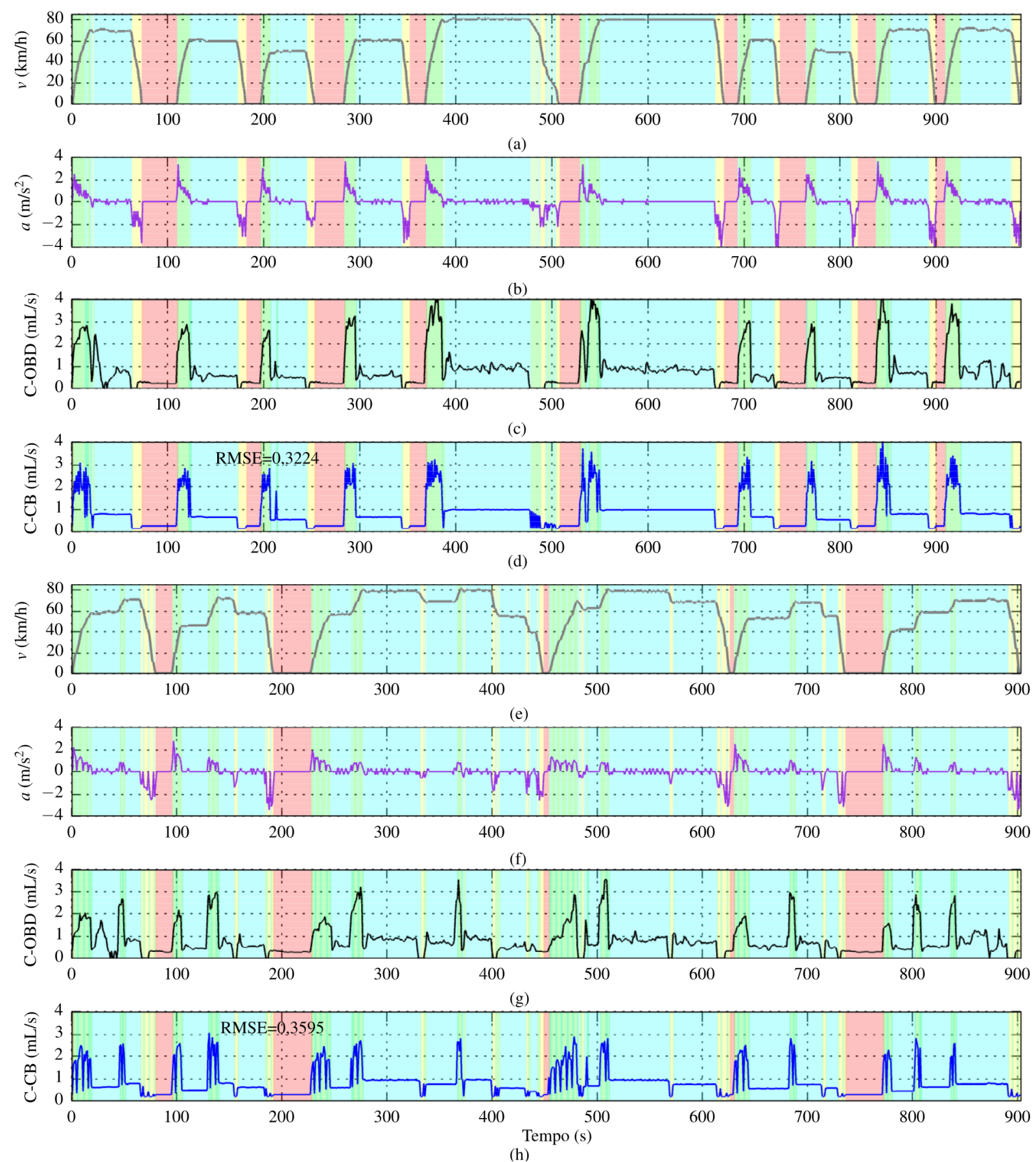

Figura 4. Perfis de velocidades, acelerações e consumos de combustível (medido e estimado) para cada cenário de tráfego

\section{ESTUDO DE CASO}

Para ilustrar a importância da calibração dos modelos de combustível na avaliação de sistemas 
de tráfego, apresenta-se estudo por simulação de mudanças de infraestrutura em uma malha viária real. Faz-se uma comparação do consumo de combustível antes e depois da mudança de infraestrutura considerando dois casos: i) os parâmetros do modelo de combustível foram mantidos nos valores padrão do simulador (TSS, 2015); ii) os parâmetros do modelo de combustível foram calibrados seguindo a abordagem propostas neste artigo. Apenas veículos de passeio são considerados. Na Tabela 1 são apresentados os valores padrão do AIMSUN e os valores obtidos da calibração de quatro veículos.

Tabela 1: Parâmetros do modelo de consumo de combustível usados na simulação

\begin{tabular}{llllll}
\hline Parâmetros & AIMSUN & Honda Civic & Ford Fiesta & VW Saveiro & Ford Fusion \\
\hline$F_{\mathrm{i}}(\mathrm{mL} / \mathrm{s})$ & 0,330 & 0,295 & 0,260 & 0,334 & 0,523 \\
$c_{1}(\mathrm{~mL} / \mathrm{s})$ & 0,420 & 1,065 & 1,403 & 1,116 & 1,930 \\
$c_{2}\left(\mathrm{~mL} \cdot \mathrm{s}^{2} / \mathrm{m}^{2}\right)$ & 0,260 & 0,095 & 0,031 & 0,061 & 0,155 \\
$F_{1}(\mathrm{~L} / 100 \mathrm{~km})$ & 4,700 & 4,690 & 5,570 & 6,190 & 10,43 \\
$F_{2}(\mathrm{~L} / 100 \mathrm{~km})$ & 6,500 & 6,250 & 7,170 & 7,290 & 12,10 \\
$v_{\mathrm{m}}(\mathrm{km} / \mathrm{h})$ & 50,00 & 56,72 & 54,15 & 64,22 & 64,42 \\
$F_{\mathrm{d}}(\mathrm{mL} / \mathrm{s})$ & 0,530 & 0,185 & 0,252 & 0,512 & 0,274 \\
\hline
\end{tabular}

\section{1. Área de estudo}

A área escolhida para o estudo corresponde a um trecho da Rua Deputado Antônio Edu Vieira em Florianópolis/SC. Essa área foi escolhida pois já se dispunha do modelo de simulação para o simulador AIMSUN calibrado para condições de tráfego vigentes. Atualmente, a Rua Deputado Antônio Edu Vieira está sendo duplicada e modificada para comportar um corredor exclusivo para transporte coletivo nos dois sentidos de circulação.

A Figura 5(a) apresenta a infraestrutura atual e a Figura 5(b) apresenta a infraestrutura que estará disponível após a duplicação e implantação do corredor exclusivo para transporte coletivo. A malha viária do cenário atual foi calibrada com dados de campo, inclusive com levantamento de interferências de pedestres e de manobras para estacionamento. Dados coletados com veículo sonda equipado com o sistema de aquisição proposto neste trabalho também foram usados para a calibração e validação da modelagem da malha viária. A calibração foi realizada para o horário de pico da manhã (das 7:00 às 9:00 h) para um dia de semana típico. Um trabalho rigoroso de modelagem e calibração foi realizado com o intuito de minimizar erros de estimação (Sbayti e Roden, 2010).

Congestionamentos na região escolhida são intensos, provocados pela capacidade viária insuficiente para a demanda em horários de pico. Agrava-se o problema pelas diversas interseções não semaforizadas com conversões à esquerda, manobras de estacionamento, circulação de pedestres, e presença de polos geradores de viagens no entorno da rede de interesse, como a Universidade Federal de Santa Catarina, Eletrosul e condomínios residenciais. 


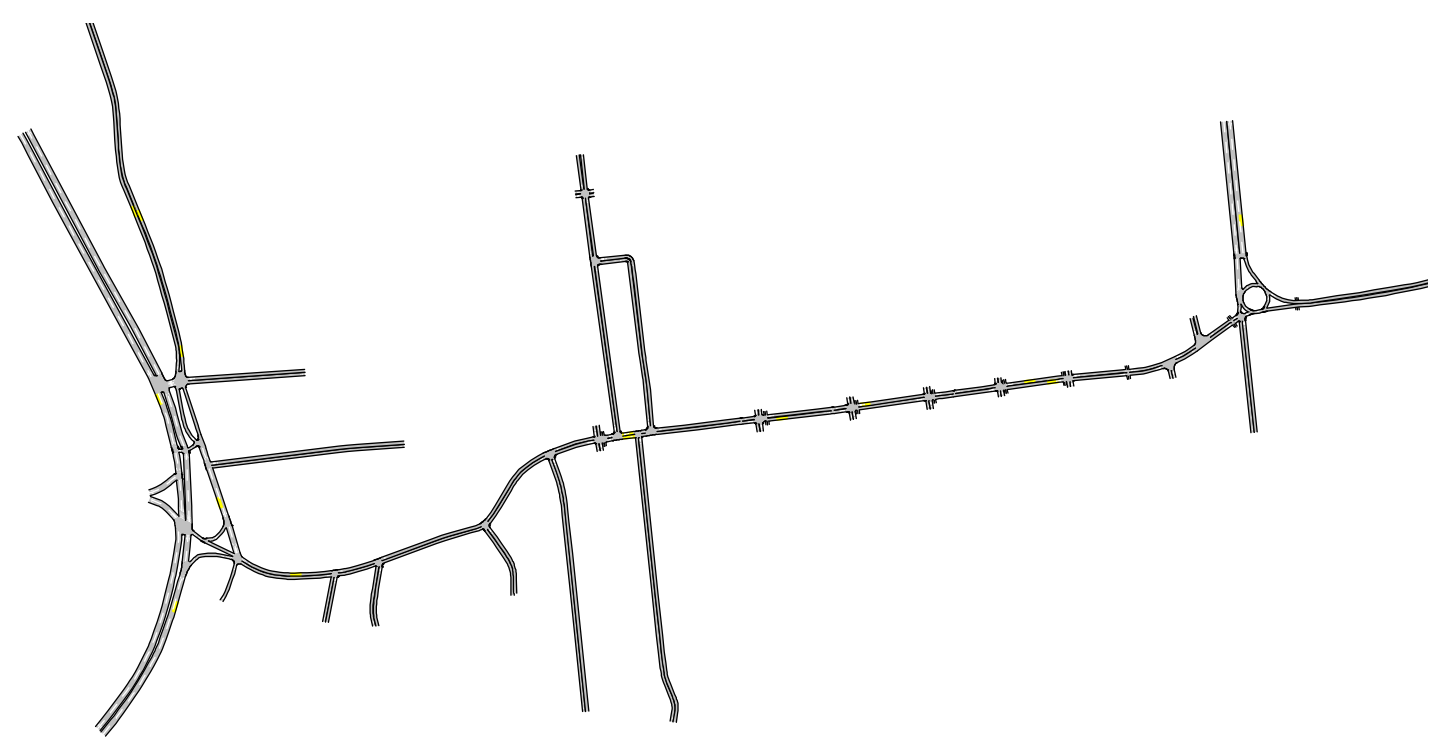

(a)

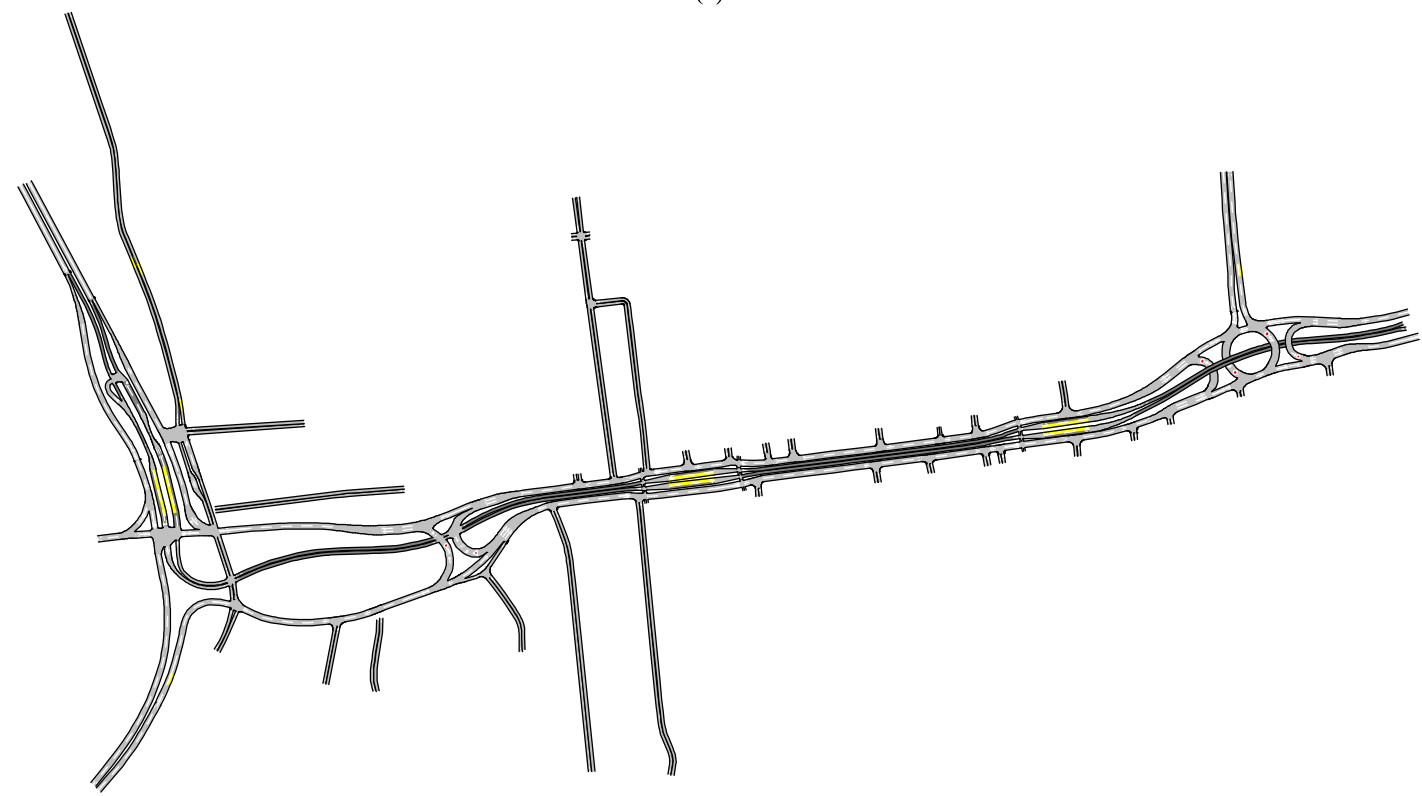

(b)

Figura 5. Rede de interesse para (a) cenário atual e (b) cenário futuro

Congestionamentos na região escolhida são intensos, provocados pela capacidade viária insuficiente para a demanda em horários de pico. Agrava-se o problema pelas diversas interseções não semaforizadas com conversões à esquerda, manobras de estacionamento, circulação de pedestres, e presença de polos geradores de viagens no entorno da rede de interesse, como a Universidade Federal de Santa Catarina, Eletrosul e condomínios residenciais.

\subsection{Composição de automóveis no tráfego}

A malha viária foi calibrada apenas com carros de passeio e ônibus. Para definir a composição do tráfego em termos dos quatro veículos de passeio para os quais foram obtidos parâmetros do modelo de combustível, foram usados dados da Anfavea (2016) do número de licenciamentos de automóveis realizados no país em 2016. Esses dados mostram 33,5\% dos automóveis novos licenciados com motorização 1.0 , 65\% com motorização até 2.0 e 1,5\% com motorização maior do que 2.0. Com base nesses dados, foram usadas as seguintes proporções do tráfego: veículos 1.0 compuseram 33,5\% do total, veículos 1.6 e 1.8 compuseram 32,5\% do total cada, e 
os veículos 2.4 compuseram 1,5\%. Ainda que esses valores não reflitam necessariamente a realidade local, permitem uma variedade significativa dos tipos de veículos de passeio. Os ônibus seguem a programação real do serviço de transporte coletivo da cidade, porém os dados de consumo de combustível não foram considerados neste trabalho ainda que seja possível a instrumentação via OBD de veículos pesados.

\subsection{Configuração}

Para cada um dos quatro cenários simulados foram realizadas vinte replicações, isto é, vinte execuções da simulação. Cada replicação é inicializada com uma semente diferente, que serve de base para o gerador de números pseudo-aleatórios que, por sua vez, produz valores estocásticos diferentes para cada execução. Os dados de consumo de combustível por faixa de motorização apresentados na Seção 5.4 correspondem à média aritmética dos resultados das 20 replicações. Já os percentuais correspondem à média das diferenças de cada replicação, a fim de garantir a diversidade de cada cenário simulado. 0 passo de simulação usado foi de $\Delta t=0,6 \mathrm{~s}$.

\subsection{Resultados}

O consumo de combustível global obtido em simulação por automóveis é apresentado na Tabela 2. No cenário atual, observou-se que a estimação do consumo de combustível global utilizando parâmetros padrão foi $25,1 \%$ maior do que a estimação com parâmetros calibrados, com sobrestimação de 321 litros. Da mesma maneira, no cenário futuro, a estimação com parâmetros padrão foi $18,8 \%$ maior do que a estimação com parâmetros calibrados, com uma sobrestimação de 172,5 litros. Os resultados mostram que existe uma diferença significativa na estimação do consumo de combustível global usando parâmetros apresentados no manual versus parâmetros obtidos da calibração com dados do OBD.

Tipicamente o que se deseja é verificar o desempenho relativo entre dois cenários. A comparação entre o cenário atual e o cenário futuro com parâmetros padrão mostra uma redução do consumo de combustível de 30,5\%. Já a redução do consumo de combustível no caso com parâmetros calibrados é de 23,7\%. Ou seja, com o uso dos parâmetros padrão há uma superestimação dos benefícios auferidos pela mudança de infraestrutura em termos de redução no consumo de combustível de aproximadamente $7 \%$.

Tabela 2: Consumo de combustível obtido em simulação por categoria de veículos

\begin{tabular}{|c|c|c|c|c|c|c|c|c|}
\hline \multirow[b]{2}{*}{$\begin{array}{c}\text { Faixa de } \\
\text { motorização }\end{array}$} & \multicolumn{3}{|c|}{ Cenário atual } & \multicolumn{3}{|c|}{ Cenário futuro } & \multicolumn{2}{|c|}{ Entre cenários } \\
\hline & $\begin{array}{l}\text { Parâmetros } \\
\text { Padrão (L) }\end{array}$ & $\begin{array}{l}\text { Parâmetros } \\
\text { Calibrados (L) }\end{array}$ & $\begin{array}{c}\text { Diferença } \\
(\%)^{*}\end{array}$ & $\begin{array}{l}\text { Parâmetros } \\
\text { Padrão (L) }\end{array}$ & $\begin{array}{l}\text { Parâmetros } \\
\text { Calibrados (L) }\end{array}$ & $\begin{array}{c}\text { Diferença } \\
(\%)^{*}\end{array}$ & $\begin{array}{l}\text { Parâmetros } \\
\text { Padrão (\%) }\end{array}$ & $\begin{array}{c}\text { Parâmetros } \\
\text { Calibrados (\%) }\end{array}$ \\
\hline 1.0 litros & 427,4 & 284,3 & $-33,5$ & 305,1 & 223,8 & $-26,6$ & $-28,4$ & $-21,0$ \\
\hline 1.6 litros & 417,5 & 341,5 & $-18,2$ & 299,3 & 261,0 & $-12,7$ & $-28,0$ & $-23,3$ \\
\hline 1.8 litros & 414,1 & 304,5 & $-26,5$ & 298,4 & 238,2 & $-20,2$ & $-27,7$ & $-21,5$ \\
\hline 2.4 litros & 22,0 & 29,7 & 35,4 & 13,5 & 20,8 & 54,4 & $-37,9$ & $-29,2$ \\
\hline Global & $1.281,0$ & 960,0 & $-25,1$ & 916,3 & 743,8 & $-18,8$ & $-30,5$ & $-23,7$ \\
\hline
\end{tabular}

*A diferença foi obtida a partir da média das diferenças de cada replicação e não a partir dos valores médios

\section{CONSIDERAÇÕES FINAIS}

O modelo de consumo de combustível do simulador AIMSUN foi calibrado usando dados de sensores automotivos e obtidos via sistema OBD embarcado em veículos. Resultados foram valida- 
dos para um ciclo de condução, apontando que o modelo é capaz de fornecer avaliações consistentes do consumo de combustível em trajetos com vários modos de condução.

Em estudo de caso de comparação de cenários de infraestrutura, a estimação do consumo de combustível com parâmetros padrão indicou uma sobrestimação nos resultados de cerca de $30 \%$ em relação à estimação com parâmetros calibrados. Além disso, a redução no consumo de combustível do cenário futuro em relação ao atual foi superestimada em 7\% com parâmetros padrão. Um dos motivos dessa sobrestimação é o fato de os parâmetros apresentados no manual terem sido obtidos para veículos da década de 1980, que historicamente consumiam mais combustível pois não possuíam injeção eletrônica de combustível. Ademais, o uso dos parâmetros padrão levou em consideração apenas um tipo de veículo. A utilização de um método de aquisição de dados, calibração, validação e implementação do modelo de consumo de combustível no simulador de tráfego, que leva em consideração dados obtidos em campo, se mostrou efetiva e satisfatória.

A mesma abordagem empregada neste trabalho pode ser empregada com outros modelos de consumo de combustível. Neste sentido, trabalhos futuros investigarão meios para estimar a emissão de poluentes e também a estimação de combustível em veículos pesados, como caminhões e ônibus.

\section{REFERENCES}

ABNT (2010) NBR 7024 - Veículos Rodoviários Automotores Leves - Medição do Consumo de Combustível - Método de Ensaio. Associação Brasileira de Normas Técnicas, Rio de Janeiro.

ABNT (2012) NBR 6601 - Veículos Rodoviários Automotores Leves - Determinação de Hidrocarbonetos, Monóxido de Carbono, Óxidos de Nitrogênio, Dióxido de Carbono e Material Particulado no Gás de Escapamento. Associação Brasileira de Normas Técnicas, Rio de Janeiro.

Ahn, K. (1998) Microscopic Fuel Consumption and Emission Modeling. Dissertação (Mestrado) - Faculty of the Virginia Polytechnic Institute and State University. Virginia, EUA.

Akçelik, R. (1983) Progress in Fuel Consumption Modelling for Urban Traffic Management. Australian Road Research Board. Report ARR 124. Vermont South, Austrália, p. 51-56. ISSN 0518-0728. URL: http://www.sidrasolutions.com/Cms_Data/Contents/SIDRA/Folders/Resources/Articles/Articles/ contents/DP42CMSR2F37U597/Akcelik_ARR124_FuelConsumption.PDF

Akçelik, R. e M. Besley (2003) Operational Cost, Fuel Consumption, and Emission Models in aaSIDRA and aaMOTION. 25th Conference of Australian Institutes of Transport Research. Adelaide, Austrália. URL: http://www.sidrasolutions.com/documents/AKCELIK_COSTModels(CAITR\%202003)v2.pdf

Alessandrini, A.; F. Filippi e F. Ortenzi (2004) Consumption Calculating of Vehicles Using OBD Data. Centre for Transprtation and Logistics. Roma. URL: https://pdfs.semanticscholar.org/997e/d8cbf734a31cf45c8143115bc0880a48ef9e.pdf

An, F.; R. Earley e L. Green-Weiskel (2011) Global Overview on Fuel Efficiency and Motor Vehicle Emission Standards: Policy Options and Perspectives for International Cooperation. Commission on Sustainable Development Report. Nova Iorque, EUA, p. 22. URL: https://cleanenergysolutions.org/es/resources/global-overview-fuel-efficiency-motor-vehicle-emissionstandards-policy-options

André, M.; R. Joumard; R. Vidon; P. Tassel e P. Perret (2005) Real-World European Driving Cycles, for Measuring Pollutant Emissions from high- and low-powered cars. Laboratory Transport and Environment, INRETS. França, v. 40, p. 5944-5953. DOI: 10.1016/j.atmosenv.2005.12.057

ANFAVEA (2016) Associação Nacional dos Fabricantes de Veículos Automotores. Estatística de produção, vendas e exportação de automóveis. São Paulo. Acesso em 10 fev. 2017. Disponível em: <http://www.anfavea.com.br/estatisticas-2016.html>.

Baltusis, P. (2004) On Board Vehicle Diagnostics. Convergence International Congress \& Exposition on Transportation Electronics. SAE Technical Paper No. 2004-21-0009. URL: https://www.sae.org/publications/technical-papers/content/2004-21$0009 /$

Barlow, T.; S. Latham; I. McCrae e P. Boulter (2009) A Reference Book of Driving Cycles for Use in the

Measurement of Road Vehicle Emissions. TRL Published Project Report. Wokingham, Reino Unido. URL: https://trl.co.uk/reports/PPR354

Chai, T. e R. Draxler (2014) Mean Square Error (RMSE) or Mean Absolute Error (MAE) - Arguments Against

Avoiding RMSE in the Literature. Geoscientific Model Development. EUA. p.1247-1250. DOI: 10.5194/gmd-7-1247-2014

CONAMA (2004) Dispõe Sobre os Requisitos para Adoção de Sistemas de Diagnose de Bordo - OBD nos Veículos Automotores Leves Objetivando Preservar a Funcionalidade dos Sistemas de Controle de Emissão. Resolução n. 354, de 13 de dezembro de 2004. Diário Oficial da República Federativa do Brasil. Brasília, n. 239. 
CIMA (2015) Dispõe Sobre Fixar o Porcentual Obrigatório de Adição de Etanol Anidro Combustível à Gasolina. Portaria n. 75, de 5 de março de 2015. Conselho Interministerial do Açúcar e do Álcool. Diário Oficial da União. Brasília, n. 44.

Ferreira, L. J. A. (1982) Car Fuel Consumption in Urban Traffic: The Results of a Survey in Leeds Using Instrumented Vehicles. Institute of Transport Studies, Universidade de Leeds, Inglaterra, p. 77. URL: http://eprints.whiterose.ac.uk/2376/

FISPQ (2014) Gasolina Especial BR E27. Ficha de Informação de Segurança de Produto Químico. Petrobrás

Distribuidora S.A. Rio de Janeiro, p.14.

Fonseca, H.; C. Ferreira e T. Fernandes (2012) New Methodologies to Measure in Real Time Fuel Consumption of Internal Combustion Engines. International Conference on Experimental Mechanics. Leiria, Portugal. URL: https://www.researchgate.net/publication/261872468

Franco, V.; M. Kousoulidou; M. Muntean; L. Ntziachristos; S. Hausberger e P. Dilara (2013) Road Vehicle Emission Factors Development: A Review. Atmospheric Environment, n.70, p.84-97. DOI: 10.1016/j.atmosenv.2013.01.006

Gomes, C. (2016) Calibração de Motores. Curso SAE Brasil. São Paulo. Acesso em: 27 jul. 2016. 316 slides. Apresentação em Power-point. Disponível em: <http://saebrasil.org.bra2016cLinkcalibracaomotores.zip>.

Hickman, A. (1988) Methodology for Calculating Transport Emissions and Energy Consumption. Transport Research Laboratory - Project Report SE/491/98. Reino Unido, p. 362. URL: https://trimis.ec.europa.eu/project/methodology-calculatingtransport-emissions-and-energy-consumption\#tab-outline

Lai, J.; L. Yu; G. Song; P. Guo e X. Chen (2013) Development of City-Specific Driving Cycles for Transit Buses Based on VSP Distributions: Case of Beijing. Journal of Transport Engineering. Pequim, China, v. 139, p. 749-757. ISSN 0733-947X. DOI: 10.1061/(ASCE)TE.1943-5436.0000547

Liu, J.; X. Wang e A. Khattak (2016) Customizing Driving Cycles to Support Vehicle Purchase and Use Decisions: Fuel Economy Estimation for Alternative Fuel Vehicle Users. Transportation Research Part C: Emerging Technologies, p. 281-298. DOI: 10.1016/j.trc.2016.02.016

Marinho, A. T. (2016) Calibração de Modelos de Consumo de Combustível para Microssimulação de Tráfego com Dados Coletados Via OBD. Dissertação (Mestrado) - PPGEAS - UFSC, Florianópolis, Brasil.

Nanduri, K. (2013) Mitigating Emissions and Energy Consumption for Urban Transportation Networks: Simulation-Based Signal Control Strategies. Dissertação (Mestrado). Massachusetts Institute of Technology. Massachussetts, USA.

Osorio, C. e K. Nanduri (2015) Energy-Efficient Urban Traffic Management: A Microscopic Simulation-Based Approach. Transportation Science. v. 49, n. 3, p 637-651. ISSN 1526-5447. DOI:10.1287/trsc.2014.0554

Owens, R. e M. Laughlin (2016) Case Study - Idling Reductions Technologies for Emergency Service Vehicles. U.S. Department of Energy. Chicago, EUA, p. 36. URL: https://anl.box.com/s/hfg7oetq9b7auer0ehz17ojf5gdvvzjv

Sbayti, H. e D. Roden (2010) Best Practices in the Use of Micro Simulation Models. Transportation Research Board Report. Arlington, EUA, p. 81. URL:

http://statewideplanning.org/wp-content/uploads/259_NCHRP-08-36-90.pdf

Thibault, L.; P. Degeilh; O. Lepreux; L. Voise; G. Alix e G. Corde (2016) A new GPS-based method to estimate real driving emissions. IEEE $19^{\text {th }}$ International Conference on Intelligent Transportation Systems (ITSC). Rueil-Malmaison, França. ISSN 2153-0017. DOI: 10.1109/ITSC.2016.7795776

TSS (2015) Manual do Usuário do Simulador AIMSUN. Transport Simulation Systems. Barcelona, Espanha, p. 393-396.

Ulsoy, G.; H. Peng e M. Çakmaci (2012) Automotive Control Systems. Cambridge University Press. New York, USA.

Watson, H. (1973) Influence of Driving Patterns on Localized Urban Emissions Source. SAE Technical Paper. n. 730556. EUA, p. 15. DOI: $10.4271 / 730556$ 